\title{
Filling the ceratosaur gap: A new ceratosaurian theropod from the Early Cretaceous of Spain
}

\author{
Bárbara Sánchez-Hernández and Michael J. Benton \\ Acta Palaeontologica Polonica 59 (3), 2014: 581-600 doi: http://dx.doi.org/10.4202/app.2011.0144
}

Ceratosaurian theropods evolved in two bursts, first in the Middle and Late Jurassic and then in the Late Cretaceous, leaving a 20 Myr gap in the Early Cretaceous during which remains are rare. We describe here a new ceratosaurian theropod, Camarillasaurus cirugedae , from fluvial deposits of the Camarillas Formation (lower Barremian, Lower Cretaceous) of Camarillas, Teruel Province, NE Spain. The new theropod is represented by a collection of associated bones, including a tooth, a possible cervical vertebra, two sternal plates, the proximal part of a right tibia, a broken right scapulocoracoid, the incomplete sacrum, five caudal vertebrae, an isolated caudal neural arch, a chevron, an almost complete presacral rib and some fragments of vertebrae, ribs, and other elements. Camarillasaurus is differentiated from other theropods by the extreme depth of the tibia proximal end, and a deep longitudinal groove on the tibia. The new dinosaur is a ceratosaur, phylogenetically close to the base of the clade, and perhaps more derived than the Chinese basal ceratosaur Limusaurus. The new taxon is significant in the evolution of the ceratosaurian dinosaurs, being placed temporally between its more common Jurassic and mid-Upper Cretaceous relatives, and it is one of only a few from Laurasia.

Key words: Dinosauria, Theropoda, Ceratosauria, Cretaceous, Teruel Province, Aragón, Spain.

Bárbara Sánchez-Hernández [bshdez@hotmail.com] and Michael J. Benton [Mike.Benton@bristol.ac.uk](corresponding author), School of Earth Sciences, University of Bristol, Queens Road, Bristol, BS8 1RJ, United Kingdom.

This is an open-access article distributed under the terms of the Creative Commons Attribution License (for details please see creativecommons.org), which permits unrestricted use, distribution, and reproduction in any medium, provided the original author and source are credited. 
PaF Full text $(1,220.1 \mathrm{kB})$ 